\title{
Blood culture: improvement project through training and feedback of results and quality criteria
}

\author{
Annamaria Calvo', Enrica Martini' ${ }^{2}$, Claudia Cutrini', Sandra Savini', Andrei Zhdan², Manso Esther' \\ I SOS Microbiologia \\ 2 Servizio di Igiene Ospedaliera Ospedali Riuniti di Ancona, Ancona
}

Key words: blood culture, quality control

Emocolture: progetto di miglioramento mediante formazione e feedback dei risultati e dei criteri di qualità

\section{SUMMARY}

Although blood culture is one of the most important tests in a microbiology laboratory, the need for its request is not always well understood by the clinician, and its execution is frequently wrong. In order to improve the quality of the application and enforcement of blood cultures, 15 training sessions for clinicians and nurses in groups of about 100 at a time with ECM were carried out. The protocol was published in the Intranet site of the ClO (Controllo Infezioni Ospedaliere). Blood cultures were subsequently performed for the departments that collected more than 50 samples/semester and provided a report every six months. This report contained the pertinent information, including the number of blood cultures that were collected from the peripheral vein, the artery or by catheter, the number of samples collected correctly (at least 2 during 48 hours) and incorrectly (single set in 48 hours), the rate of contamination and the percentage of positive cultures. When the indexes were inconsistent with the established quality criteria, a warning signal was issued. In the case of too large inconsistencies, the report was handed directly to the Head of the department, with whom the possible causes of inadequacy and how to correct them were discussed. In this work we propose this system aimed at improving the quality of blood cultures.

L'emocoltura è uno degli esami più importanti in un Laboratorio di Microbiologia, tuttavia la sua richiesta non sempre è ben compresa dal medico e la sua esecuzione è spesso errata. Gli errori si riscontrano sia nel numero di campioni prelevati per episodio infettivo che per il sito di prelievo dell'emocoltura. Con questo lavoro si mette in luce l'importanza di istituire corsi ad hoc sulle emocolture, rivolti sia a medici sia ad infermieri e di realizzare report, contenenti dati feedback e livello di qualità delle emocolture, specifici per reparto, La consegna dei dati ai reparti e la discussione dei risultati critici con i capi reparto hanno l'obiettivo di migliorare la qualità delle emocolture.

Sono stati organizzati in ospedale corsi ECM sull'emocoltura della durata di un giorno frequentati da cento uditori per ogni giornata in sessioni differenti per medici (3 gg) e per infermieri (12 gg).

Ad ogni reparto è stato consegnato un poster esplicativo delle procedure di prelievo per l'emocoltura (1). In dettaglio le vari fasi comprendono $(3,4)$ : la preparazione dei flaconi, quella dell'operatore, l'antisepsi della cute (7), il prelievo (6) e l'inoculazione dei flaconi. In pratica: la corretta preparazione dei flaconi prevede di fare a) un segno sui flaconi per non superare la quantità di sangue raccomandata, b) rimozione dei tappi, c) disinfezione con clorexidina 2\% (un tampone per ogni flacone), d) lasciare asciugare per $1 \mathrm{~min}$. L'operatore ha l'obbligo di: a) indossare la maschera facciale, b) procedere ad un lavaggio antisettico delle mani.

Per eseguire il prelievo in modo asettico l'operatore applica un laccio emostatico, sceglie il sito di prelievo, toglie il laccio emostatico, quindi disinfetta il sito prescelto con garze sterili e clorexidina $2 \%$ (eseguendo movimenti circolari dal centro alla periferia), l’operazione va ripetuta. Per il prelievo si devono indossare guanti non sterili puliti, si inserisce l'ago nella vena e si aspira lentamente la quantità di sangue prevista e riassunta nella Tabella 1 . Si procede pertanto all'inoculazione dei falconi per aerobi ed anaerobi (Tabella I). Dopo l'inoculazione dei flaconi si miscela il contenuto per evitare coaguli, si disinfetta il tappo dei flaconi, si eliminano aghi e butterfly nell'ago-box, quindi si tolgono i guanti.

Si applica sui flaconi l'etichetta senza coprire il codice a barre e si registra l'ora del prelievo. Si procede quindi al lavaggio antisettico delle mani.

Nel poster consegnato ai reparti è segnalato anche il "momento del prelievo", il sito del prelievo e il numero di prelievi che è opportuno eseguire al fine di una corretta valutazione finale (2).

Momento del prelievo: il primo deve essere effettuato all’inizio del rialzo febbrile, il secondo dopo 15-30 minuti, possibilmente prima dell'inizio della terapia antibiotica. Nel paziente già in terapia antibiotica il prelievo di sangue va effettuato subito prima della somministrazione della dose successiva di antibiotici.

Il sito del prelievo è una vena periferica, scegliendo vene diverse per ciascun prelievo. Se il paziente è portatore di catetere vascolare si deve associare l'emocoltura da catetere vascolare con un'altra da vena periferica.

Il numero di prelievi è stabilito in due/tre per episodio febbrile, da vene periferiche diverse, tenendo presente che un prelievo consta di un set di flaconi (anaerobi, aerobi) (5).

Sono stati adottati dei criteri denominati "Indicatori di qualità” che permettono di avere un controllo sulla validità del saggio eseguito, in particolare il tasso di contaminazione

Tabella I.

\begin{tabular}{lccc}
\hline PESO & DANGUE DA PRELEVARE & SANGUE DA INOCULARE & TIPO DI FLACONE \\
\hline Peso $<\mathrm{Kg} 8$ & $\mathrm{I} \mathrm{ml}$ & $\mathrm{I} \mathrm{ml}$ - Pediatrico (Aerobi/Miceti) & Giallo \\
\hline Peso $>\mathrm{Kg} 8<\mathrm{Kg} \mathrm{l} 3$ & $3 \mathrm{ml}$ & $3 \mathrm{ml}-$ Pediatrico (Aerobi/Miceti) & Giallo \\
\hline Peso $>\mathrm{Kg} \mathrm{I}<\mathrm{Kg} \mathrm{27}$ & $5 \mathrm{ml}$ & $5 \mathrm{ml}$ Aerobi/Miceti & Verde \\
\hline Peso $>\mathrm{Kg} 27<\mathrm{Kg} \mathrm{40}$ & $10 \mathrm{ml}$ & $10 \mathrm{ml}$ Aerobi/Miceti & Verde \\
\hline Peso $>\mathrm{Kg} 40<\mathrm{Kg} 54$ & $15 \mathrm{ml}$ & $10 \mathrm{ml}$ Aerobi/Miceti & Verde \\
& & $5 \mathrm{ml}$ Anaerobi & Arancio \\
\hline Adulti e Bambini $>\mathrm{Kg} 54$ & $20 \mathrm{ml}$ & $10 \mathrm{ml}$ Aerobi/Miceti & Verde \\
& & $10 \mathrm{ml}$ Anaerobi & Arancio \\
\hline
\end{tabular}

\section{Corresponding author: Annamaria Calvo}

S.O. Laboratorio analisi, Microbiologia, A.O. Ospedali Riuniti di Ancona

60020 Torrette (AN) - Via Conca, 6I - Tel. 07I 5964250

E-mail: annycalvo@libero.it 
deve essere inferiore al 3\% del totale degli esami eseguiti, il rapporto emocolture positive/emocolture totali deve rientrare in un intervallo compreso indicativamente tra il 6\% e il $12 \%$. Quando la percentuale di positività del campione è inferiore al 6\%, ciò è indice di un probabile eccessivo campionamento e di esecuzione di emocolture, anche in assenza di indicazione clinica. Se la positività del campione è superiore a $12 \%$ del totale, questo parametro potrebbe riflettere una situazione di scarso campionamento rispetto all'effettiva necessità. Il numero di emocolture non correttamente eseguite (prelievo unico) dovrebbe tendere a zero. Il sito del prelievo è di preferenza vena periferica (VP) non da catetere venoso centrale (CVC) tranne che in casi particolari.

L'intera procedura qui descritta è stata inserita in modo ampliato in INTRANET come linea guida sulle "modalità di esecuzione ed interpretazione delle emocolture”.

La Figura I rappresenta il documento cartaceo inviato come report ai reparti.

Figura I. Esempio di report

\begin{tabular}{|c|c|c|c|c|c|}
\hline & & $2^{\circ}$ sem 2008 & \multicolumn{2}{|c|}{$I^{\circ} \operatorname{sem} 2009$} & INDICATORI DI QUALITÀ \\
\hline EMOCOLTURE TOTALI & & 315 & \multicolumn{2}{|c|}{248} & \\
\hline \multicolumn{6}{|c|}{ CORRETTAMENTE ESEGUITE * } \\
\hline POSITIVE & & 53 & & & \\
\hline NEGATIVE & & 239 & & & \\
\hline CONTAMINATE & & $15(4.8 \%)$ & & & Tasso di contaminazione: $<3 \%$ \\
\hline TOTALI & & 307 & & & \\
\hline \multicolumn{6}{|c|}{ NON CORRETTAMENTE ESEGUITE ** } \\
\hline POSITIVE & & 0 & & & \\
\hline NEGATIVE & & 7 & & & \\
\hline NON INTERPRETABILI & & 1 & & & \\
\hline \multirow[t]{2}{*}{ TOTALI } & & 8 & & & $\begin{array}{l}\text { II numero di emocolture non } \\
\text { correttamente eseguite (prelievo } \\
\text { unico) dovrebbe tendere allo zero }\end{array}$ \\
\hline & $\mathbf{N}^{\circ}$ & $\%$ & $\mathbf{N}^{\circ}$ & $\%$ & \\
\hline \multicolumn{6}{|l|}{ CONTAMINATE + } \\
\hline NON INTERPRETABILI & 16 & 5.7 & 13 & 5.24 & \\
\hline POSITIVE/TOTALI & $53 / 315$ & 17 & $33 / 248$ & 13.3 & $\begin{array}{l}\text { Rapporto emocolture positive/totali: } \\
\text { indicativamente tra il } 6 \text { e il } 12 \% \text {. Se } \\
\text { inferiore al } 6 \% \text {, probabilmente si } \\
\text { fanno troppe emocolture, anche } \\
\text { quando non c'è indicazione. Se } \\
\text { superiore al } 12 \% \text { probabilmente se } \\
\text { ne fanno troppo poche. }\end{array}$ \\
\hline \multirow[t]{2}{*}{ SITO DI PRELIEVO } & & $2^{\circ}$ SEM 2008 & \multicolumn{2}{|c|}{$I^{\circ}$ SEM 2009} & \\
\hline & & $\mathbf{N}^{\circ}(\%)$ & \multicolumn{2}{|c|}{$\mathbf{N}^{\circ}(\%)$} & \\
\hline VENA PERIFERICA & & $158(50.2)$ & \multicolumn{2}{|c|}{$192(77.4)$} & \\
\hline $\mathrm{CVC}$ & & $157(49.8)$ & \multicolumn{2}{|c|}{$56(22.6)$} & \\
\hline ARTERIA & & 0 & \multicolumn{2}{|c|}{0} & \\
\hline CATETERE ARTERIOSO & & 0 & \multirow{2}{*}{\multicolumn{2}{|c|}{0}} & \\
\hline Consumo Citroclorex $\left(\mathrm{n}^{\circ}\right.$ & & & & & \\
\hline
\end{tabular}

\section{LEGENDA}

* CORRETTAMENTE ESEGUITE se presenti almeno 2 prelievi per lo stesso episodio febbrile nell'arco delle 24-48 ore

** NON CORRETTAMENTE ESEGUITE se presente un prelievo unico

POSITIVE:

isolamento di germe patogeno

NEGATIVE:

assenza di crescita

CONTAMINATE:

presenza di un germe commensale della cute in una sola delle 2 o più emocolture effettuate per lo stesso episodio febbrile. Dipende principalmente dalla non rigorosa antisepsi cutanea.

NON INTERPRETABILI:

presenza di un germe commensale della cute in una emocoltura quando è stato effettuato un unico prelievo

CRITICITÀ RILEVATE

AZIONI CORRETTIVE CONSIGLIATE
Permane il tasso di contaminazione superiore a quanto raccomandato $(4.55 \%)$. II numero di emocolture non correttamente eseguite (prelievo unico) è $2.42 \%$ Effettuare sempre una rigorosa antisepsi cutanea prima de prelievo Effettuare sempre almeno 2, preferibilmente 3 emocolture per episodio febbrile, in quanto le emocolture singole aumentano la probabilità di un risultato non interpretabile

In miglioramento la scelta del sito di prelievo: vengono effettuati un maggior numero di prelievi da vena periferica piuttosto che da CVC. 
I risultati di tutti i reparti sono stati raggruppati per semestre per valutare l'andamento complessivo della qualità delle emocolture nell'ospedale (Tabella 2).

Tabella 2. Valutazione della qualità di 6952 emocolture pervenute nel II semestre del 2008 e nel I semestre del 2009 al nostro laboratorio.

\begin{tabular}{|c|c|c|c|c|c|c|c|c|c|c|c|c|c|c|}
\hline & \multicolumn{5}{|c|}{2 o più 12 GG } & \multicolumn{4}{|c|}{ Una sola IN 2 GG } & \multicolumn{3}{|c|}{ TOT $D+1 D+1 \%$} & \multirow{2}{*}{ w } & \\
\hline PARTI & POS & NEG & CONT. & \%CONT. & Totale & POS & NEG & NONINT. & Totale & EMO & $\%$ & VP & & \\
\hline & 41 & 236 & & & & 53 & 513 & & 34 & & & & & \\
\hline TALE $1^{\circ}$ & 391 & 2354 & 128 & $4,46 \%$ & 2873 & 46 & 454 & 38 & 538 & 3411 & 166 & 2881 & 491 & 59 \\
\hline
\end{tabular}

Confrontando i due semestri, tra cui sono intercorsi gli aggiornamenti del personale con corsi ECM ed i report dei dati di qualità, si è riscontrato:

- Un netto miglioramento del sito di prelievo

- Una diminuzione delle emocolture non corrette

- Nessun cambiamento significativo nella percentuale di contaminazione

Il lavoro ha permesso ai responsabili di reparto di prendere coscienza del livello di qualità dell'emocoltura nella propria realtà e di apportare modifiche laddove possibile. Si ha il riscontro di aumento della qualità sia per singolo reparto sia per il totale andamento. L'unione tra la formazione attiva con corsi ECM e i report dei feedback dei risultati e dei criteri di qualità ha migliorato l'esecuzione dell'emocoltura. Il lavoro deve essere continuativo nel tempo per avere sempre più adesione ai criteri di qualità. Quindi proponiamo di adottare questo modello operativo per sorvegliare la qualità delle emocolture.

\section{BIBLIOGRAFIA}

1. Clinical and Laboratory Standard Institute. Principles and procedures for blood cultures; approved guideline. Clinical and Laboratory Standard Institute 2007.

2. Cockerill FR, III, Wilson JW, Vetter EA, et al. Optimal testing parameters for blood cultures. Clin Infect Dis. 2004; 38: 1724-30.

3. Dellinger RP, Levy MM, et al. For the International Surviving Sepsis Campaign Guidelines Committee. Surviving Sepsis Campaign: International guidelines for management of severe sepsis and septic shock. Crit Care Med, 2008; 36: 293-327.

4. Isenberg HD. Aerobic Bacteriology. Blood cultures. In Clinical Microbiology Procedures Handbook. ASM Press, Washington DC, 2004.

5. Lee A, Mirrett S, Reller B, Weistein MP. Detection of bloodstream infection in adults: how many blood cultures are need? J Clin Microbiol, 2007; 45: 3546-8.

6. Madeo M, Jackson T, Williams C. Simple measure to reduce the rate of contamination of blood cultures in accident and emergency. Emergency Medicine Journal 2005; 22: 810-1.

7. Malani A, Trimble K, Parek V, et al. Rewiev of clinical trials of skin antiseptic agents used to reduce blood colture contamination. Infection Control \& Hospital Epidemiology 2007; 28: 892-5. 